\title{
Evaluation of dual-labeled fluorescent DNA probe purity versus performance in real-time $\mathbf{P C R}$
}

\author{
Anthony T. Yeung ${ }^{1}$, Brian P. Holloway ${ }^{2}$, Pamela Scott Adams ${ }^{3}$, and Gregory L. Shipley ${ }^{4}$
}

BioTechniques 36:266-275 (February 2004)

\begin{abstract}
Real-time PCR technology using dual-labeled fluorescent oligonucleotide probes allows for sensitive, specific, and quantitative determination of mRNA or DNA targets. Historically, dual-labeled probes have been the most expensive reagent in real-time PCR because of the postsynthesis high-performance liquid chromatography (HPLC) and/or gel purification steps required due to limitations in traditional synthesis chemistry. The recent availability of quencher reagents that allow the 3' quencher incorporation as part of the on-machine synthesis has presented the possibility that probes, when carefully synthesized, may be used without extensive postsynthesis purification. This would substantially reduce cost, making the synthesis of dual-labeled fluorescent probes affordable to any DNA synthesis laboratory. The Nucleic Acids Research Group (NARG) of the Association of Biomolecular Resource Facilities (ABRF) (Santa Fe, NM, USA) tested the hypothesis that now any DNA synthesis laboratory is capable of making quality dual-labeled fluorescent probes suitable for real-time PCRs without the need for postsynthesis purification. Members of the DNA synthesis community synthesized dual-labeled human $\beta$-actin probes and submitted them for quality and functional analysis. We found that probes that were at least $20 \%$ pure had the same efficiency as those near $100 \%$ purity, but the sensitivity of the assay was reduced as the level of purity decreased.
\end{abstract}

\section{INTRODUCTION}

The quantification of nucleic acids has evolved from Southern and Northern blot analysis to RNase-protection assays to competitive reverse transcription PCR (RT-PCR). At each step in this evolution, the required amount of template was reduced, the degree of accuracy was enhanced, and the throughput was increased. Currently, the state of the art is real-time PCR (1-3), which allows the quantification of nucleic acids at low template copy numbers with a high degree of specificity and accuracy. Although there are many fluorescence-based assay systems currently available for real-time PCR, the highest template specificity comes from those utilizing a dual-labeled fluorescent oligonucleotide probe. Intact probes have low signal from the reporter dye because of fluorescence resonance energy transfer (FRET) (4). Upon cleavage by the 5'-nucleotidase activity of Taq DNA polymerase (5), FRET is lost, and a full signal is realized from the reporter. Some of the current uses for real-time PCR include the validation of findings from microarrays, zygosity testing of transgenic animals, determination of bacterial or viral loads, and transcript quantification (6,7).

Real-time PCR instrumentation has been available since 1996. However, the high cost of dual-labeled FRET probes, resulting from the difficulties encountered during synthesis and purification, has kept this technique from reaching its full potential. Traditionally, dual-labeled FRET probes were synthesized with a $3^{\prime}$ amino group and a $5^{\prime}$ reporter dye. A $3^{\prime}$ quencher molecule was added postsynthesis by coupling an active ester to the $3^{\prime}$ amino group. To separate unreacted dye and obtain a pure full-length dual-labeled probe, two cycles of high-performance liquid chromatography (HPLC) purification and/or polyacrylamide gel electrophoresis (PAGE) purification were employed. Postsynthesis purifi- cation steps as well as the difficulties encountered during chemical synthesis have been the major factors contributing to the high cost of these probes. The recent availability of $3^{\prime}$ quencher controlled pore glass (CPG) synthesis support columns simplifies the synthesis procedure and may alleviate the need for postsynthesis purification if the oligonucleotide synthesis is of high quality. This would substantially reduce the cost, making the synthesis of real-time PCR probes affordable to any DNA synthesis laboratory. The Nucleic Acids Research Group (NARG) of the Association of Biomolecular Resources Facilities (ABRF) (Santa Fe, NM, USA) has tested the hypothesis that now any DNA synthesis laboratory is capable of making quality dual-labeled fluorescent probes suitable for real-time PCRs without the need for postsynthesis purification. We invited all DNA synthesis laboratories to synthesize dual-labeled FRET probe(s) and to submit them for analytical and

${ }^{1}$ Fox Chase Cancer Center, Philadelphia, PA, ${ }^{2}$ Centers for Disease Control and Prevention, Atlanta, GA, ${ }^{3}$ Trudeau Institute, Saranac, NY, ${ }^{4}$ University of Texas Health Science Center-Houston, Houston, TX, USA 
functional analysis. We compared a new variety of nonfluorescent quencher, the Black Hole Quencher 1 (BHQ-1 ${ }^{\mathrm{TM}}$; Biosearch Technologies, Novato, CA, USA), versus 6-carboxy$\mathrm{N}, \mathrm{N}, \mathrm{N}^{\prime}, \mathrm{N}^{\prime}$-tetramethylrhodamine (TAMRA ${ }^{\text {TM; }}$ Glen Research, Sterling, VA, USA), a fluorescent dye that has historically been used for quenching the carboxyfluorescein (FAM) reporter. The BHQ-1 quencher has no intrinsic background fluorescence and requires no changes to standard oligonucleotide synthesis procedures, making it possible to complete the synthesis and deprotection of a dual-labeled FRET probe on a standard automated DNA synthesizer. In contrast, a nonstandard mild deprotection protocol is required for TAMRA-quenched probes. Constituent academic core facilities that do not routinely make fluorescent probes were especially encouraged to synthesize a probe. Here we report the analytical and functional test results of our study.

\section{MATERIALS AND METHODS}

\section{Study Participation and Data Acquisition}

Participants for this study were recruited by invitation through list servers and individual e-mails. Potential participants were directed to a web site where they could read about the study and learn how to participate. Protocols, examples of quality analyses, and a tutorial on probe synthesis were available online. Participants were asked to synthesize a specific 5' FAM-labeled human $\beta$-actin probe. The choice of $3^{\prime}$ quencher was left to the individual respondent. Additionally, they were asked to submit a sample survey sheet defining the conditions, reagents, etc., which were used to synthesize the probe. Samples were submitted so as to assure anonymity.

\section{Quality Analysis of Dual-Labeled Probes}

PAGE analysis of probe quality was performed by running $7 \mathrm{M}$ urea, $20 \%$ polyacrylamide, Tris-borate-EDTA (TBE) gels on a PROTEAN II ${ }^{\circledR}$ appa- ratus (Bio-Rad Laboratories, Hercules, CA, USA). One microliter of sample probe with $2 \mu \mathrm{L}$ loading buffer and 10 $\mu \mathrm{L}$ deionized formamide were loaded into each well. Gels were run on $0.5 \times$ TBE, $\mathrm{pH} 8.3$, at $300 \mathrm{~V}$ for 3-4 h. The products were visualized by exciting the 6-FAM reporter with $300 \mathrm{~nm}$ UV light or by staining with Stains-All ${ }^{\mathrm{TM}}$ (Sigma, St. Louis, MO, USA). Capillary electrophoresis analysis was performed using a PACE ${ }^{\mathrm{TM}}$ MDQ system (Beckman Coulter, Fullerton, CA, USA). Capillary, buffer, and gel were used as described in the eCAPTM ssDNA 100-R kit (Beckman Coulter). The samples were run using normal polarity and a capillary temperature of $30^{\circ} \mathrm{C}$ and prepared for capillary electrophoresis by adding $200 \mu \mathrm{L}$ water to $1 \mu \mathrm{L}$ dual-labeled probe (at $100 \mu \mathrm{M}$ ) in a 96-well polystyrene plate. The run time was 10 min per sample injection. Denaturing high-performance liquid chromatography (DHPLC) analyses were performed on a WAVE ${ }^{\circledR}$ HT (high-throughput) system using a DNASep ${ }^{\circledR}$ cartridge run at $80^{\circ} \mathrm{C}$ (both from Transgenomic, San Jose, CA, USA). Elution was performed with a gradient of $3 \%-20 \%$ acetonitrile in triethylammonium acetate (TEAA) buffer. Matrix-assisted laser desorption ionization-time of flight mass spectrometry (MALDI-TOF MS) analysis of the oligonucleotides (8) was performed by mixing $1 \mu \mathrm{L}$ of oligonucleotide in TE buffer (10 mM Tris, $\mathrm{pH} 7.5$, $1 \mathrm{mM}$ EDTA) with $8 \mu \mathrm{L}$ matrix solution [1:1 mixture of 2,4,6-trihydroxyacetophenone (THAP), $80 \mathrm{mg} / \mathrm{mL}$ in ethanol and ammonium citrate, $15 \mathrm{mg} /$ $\mathrm{mL}$ in $30 \%$ actetonitrile $/ 0.1 \%$ trifluoroacetic acid]. One microliter of this solution was spotted for analysis. Data were acquired on a Reflex IV MALDITOF (Bruker Daltonics, Billerica, MA, USA) in positive linear mode using FlexControl $^{\mathrm{TM}}$ acquisition software (Bruker Daltonics). Mass spectrometry data were processed using XMass ${ }^{\mathrm{TM}}$ software (Bruker Daltonics).

\section{Real-Time PCR Evaluation of Dual-Labeled Probes}

The probes were assayed for functionality by real-time PCR utilizing a human $\beta$-actin $\left(\right.$ GenBank $^{\circledR}$ accession 
no. NM_001101) assay designed by the Quantitative Genomics Core Laboratory (QGCL) at The University of Texas Health Science Center-Houston (Houston, TX, USA). The forward primer used was 997(+) 5'-CCCTGGCACCCAGCAC-3', the reverse primer was 1067(-) 5'-GCCGATCCACACGGAGTAC- $3^{\prime}$, and the TaqMan ${ }^{\circledR}$ probe (Applied Biosystems, Foster City, CA, USA) was $1020(+)$ 5'-FAM-ATCAAGATCATTGCTCCTCCTGAGCGC-3' quencher. The template was a singlestranded synthetic oligonucleotide 71-mer, 5'-CCCTGGCACCCAGCACAATGAAGATCAAGATCATTGCTCCTCCTGAGCGCAAGTACTCCGTGTGGATCGGC-3', bearing the complete amplification product for the human $\beta$-actin assay. For PCR, we used $400 \mathrm{nM}$ primers, $100 \mathrm{nM}$ probe, and $2 \times$ TaqMan Universal PCR Master Mix without Amperase ${ }^{\circledR}$ uracil-Nglycosylase (UNG) (5 $\mathrm{mM} \mathrm{MgCl}_{2}$ ) (Applied Biosystems). Assays were assembled using a Biomek ${ }^{\circledR} 2000$ robotic workstation (Beckman Coulter) for $2 \times$ master mixture plus the primers. A Genesis ${ }^{\circledR} 100$ robotic workstation (Tecan, Research Triangle Park, NC, USA) was used to add dilutions of the synthetic oligonucleotide standard or $50 \mathrm{ng}$ human genomic DNA (BD Biosciences Clontech, Palo Alto, CA, USA) per duplicate reaction. Standard curves were made by serial dilution of the single-stranded synthetic template in $100 \mathrm{ng} / \mu \mathrm{L}$ yeast tRNA (Invitrogen, Carlsbad, CA, USA). We performed the assays using an ABI PRISM ${ }^{\circledR} 7700$ Sequence Detection System (Applied Biosystems). The cycling conditions were $50^{\circ}$ for $2 \mathrm{~min}, 95^{\circ}$ for $10 \mathrm{~min}$, and 40 cycles of $95^{\circ}$ for $15 \mathrm{~s}$ and $60^{\circ} \mathrm{C}$ for 1 $\mathrm{min}$. The reporter signal was normalized to the emission of an internal reference dye carboxy-X-rhodamine (ROX ${ }^{\mathrm{TM}}$; Applied Biosystems) and plotted as the normalized reporter signal minus background $(\Delta \mathrm{Rn})$ against the number of cycles. The cycle threshold $\left(\mathrm{C}_{\mathrm{t}}\right)$ was determined for each amplification plot by setting a fixed threshold above the baseline. We compared probe performance by examining $\mathrm{C}_{t} \mathrm{~s}$ and the mean $\Delta \mathrm{Rn}$ of the standard curve values and by quantifying the slope, y-intercept, and correlation coefficient $\left(r^{2}\right)$ values of the standard curves. Mean $C_{t}$ and
$\Delta \mathrm{Rn}$ values were also determined for the 50 ng genomic DNA samples. Each data point was performed in duplicate. Although most values of duplicates are superimposable because of the use of robotics, averaged values are used in this study.

\section{RESULTS}

Eighteen laboratories participated in the study, eight of which do not routinely synthesize this type of DNA. The total number of $\beta$-actin probes submitted was 35 . Notably, laboratories that do not routinely synthesize FRET probes made 15 of the submissions. Sixteen probes were purified by a variety of methods [ 9 by HPLC, 1 by PAGE, 6 by Oligonucleotide Purification Column (OPCTM; Applied Biosystems)], and 19 were unpurified (crude) products. For the purpose of this study, "desalted-only" samples were considered crude probes. Several methods of analysis were used, including capillary electrophoresis, DHPLC, and PAGE, which were performed on all samples, and mass spectrometry, which was performed on selected samples to resolve ambiguities. The advantages of capillary electrophoresis are speed, automation, high resolution, and peak integration to determine the degree of purity. DHPLC allows for automation, resolution according to hydrophobicity, peak integration, simultaneous monitoring of UV absorption and fluorescence, plus sample collection that is not possible with capillary electrophoresis. Mass spectrometry allows for the precise determination of molecular weight. PAGE analysis is the most economical and easily distinguishes the quality of the probes by allowing visualization of full-length products and incomplete or degraded (e.g., $n$-1, 2, 3 ...) syntheses.

All 35 of the probes used $5^{\prime}$ FAM as the reporter dye. Of these probes, 23 used BHQ-1, 11 used TAM- RA, and 1 used QSY-7 ${ }^{\circledR}$ (Molecular Probes, Eugene, OR, USA) as 3' quencher dyes. All probes except NARG 10 (QSY-7), NARG 20 (TAMRA), and NARG 33 (TAMRA) (ABRF) appeared to have been made by having the quencher immobilized on the $3^{\prime}$ synthesis support column, as evidenced by the difference in mobility of the largest molecular weight band following PAGE analysis (Figure 1). Postsynthesis labeling with dye-N-hydroxysuccinmidyl (NHS) esters resulted in a slightly longer oligonucleotide because of the additional mass from an amino-linker modification necessary for coupling of the dye quencher. These size differences were also detectable by the DHPLC, capillary electrophoresis, and mass spectrometry analyses (data not shown). There are four probable postsynthesis products: $(i)$ full-length probe with reporter and quencher; (ii) failure probe with quencher but no reporter; (iii) failure probe with reporter minus quencher; and (iv), a mixture of failure $\mathrm{x}$-mer products. Integration of the areas under the peaks in the capillary electrophoresis chromatograms showed

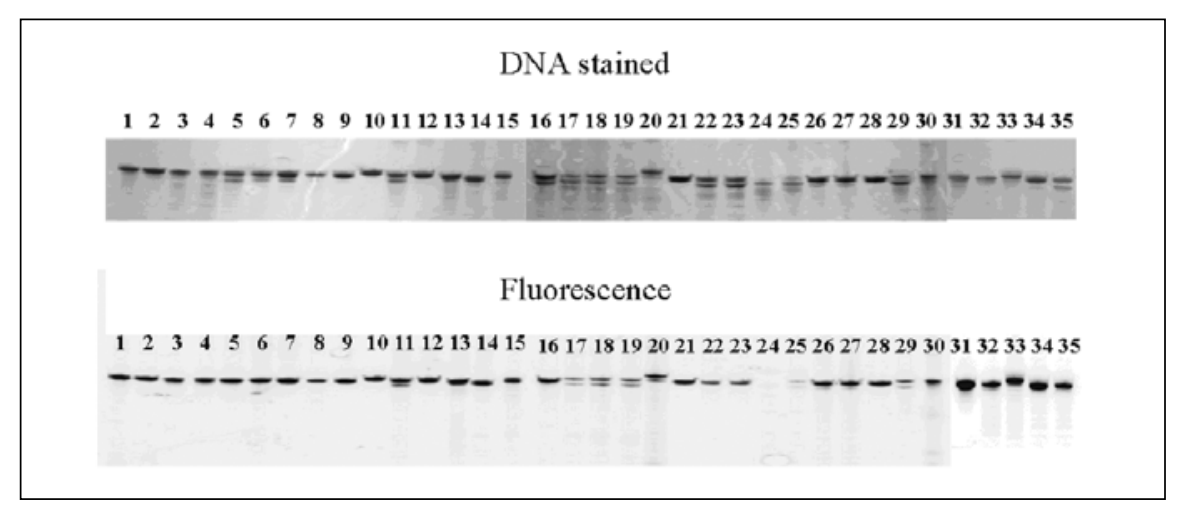

Figure 1. Polyacrylamide gel electrophoresis (PAGE) analysis of dual-labeled fluorescence resonance energy transfer (FRET) probes. Gels shown in the top panel were stained with Stains-All. All products from the syntheses were visualized. The gels shown in the bottom panel were visualized with a UV light that showed only those fragments containing fluorescent moieties. The lane numbers refer to the corresponding Nucleic Acids Research Group (NARG) probe numbers. Lanes 1-15, 16-30, and 31-35 represent electrophoresis in separate gels. 
Table 1. Summary of the Analyses for Submitted Probes Sorted by Quencher and Purity as Assessed by Capillary Electrophoresis

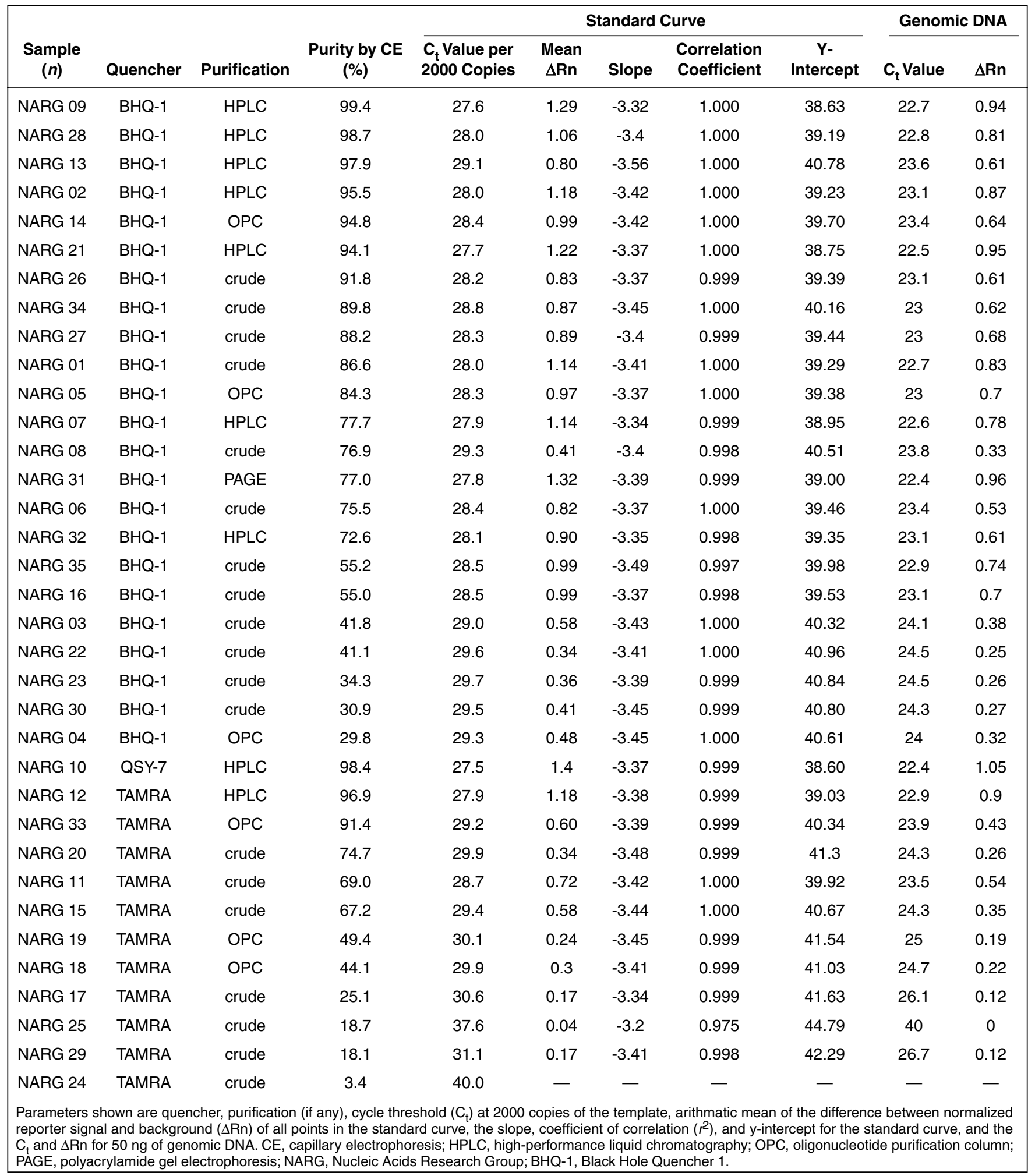

that the amounts of full-length probe ranged from $99 \%$ to $3 \%$ (Table 1). This distinction can also be easily visualized for all 35 probe samples by band density in the PAGE analyses (Figure 1) or by using capillary electrophoresis and DHPLC (Figures 2 and 3). Figure 1 shows two kinds of significant $n$ - 1 sequences: those that appear only in the DNA-stained gel (samples 5-7, 16, 22, 23, and 35) and those that appear both in the DNA-stained gel and under 
fluorescent light (NARG 11, 17-20, 25-27, 29, and 30). The syntheses in which the $n$ - 1 (lower) band is nonfluorescent and only appears on the DNAstained gel are all identified as BHQ-1quenched probes (Table 1) and can be explained by low coupling efficiency of the fluorescent reporter, resulting in a significant amount of probe with a nonfluorescent quencher (BHQ-1) but no fluorescent reporter. Where the $n-1$ band is fluorescent, most of these probes are identified as being TAMRA-quenched. Inefficient reporter coupling and the intrinsic fluorescence of TAMRA can explain the $n-1$ band. However, NARG 26, 27, and 30 are quenched by BHQ-1 and can only be explained by a product containing a reporter (FAM) but no quencher. This condition can occur when there is degradation of the full-length product from the $3^{\prime}$ end. Capillary electrophoresis analysis allows for separation of the full-length probe from failure products based on size. Figure 2 illustrates the sample profiles obtained with purified high-quality and lower-quality crude probes for both BHQ-1- and TAMRAquenched probes. Figure 2, $\mathrm{C}$ and $\mathrm{F}$

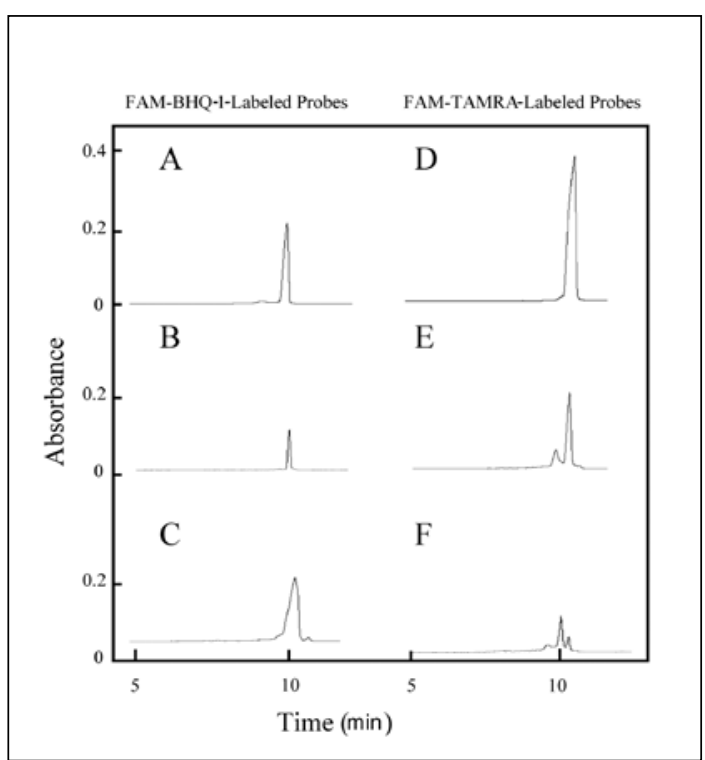

Figure 2. Resolution of probes by size utilizing capillary electrophoresis. Partially conjugated probes and/or those less than full-length came off first, followed by full-length probes with both reporter and quencher dyes. (A-C) BHQ-1-quenched probes. (D-F) TAMRA-quenched probes. (A) NARG 21, 2× high-performance liquid chromatography (HPLC)-purified. (B) NARG 01: crude. (C) NARG 06: crude of lower quality. (D) NARG 12: 1× HPLC-purified. (E) NARG 11: crude. (F) NARG 25: crude, essentially failed synthesis. BHQ-1, Black Hole Quencher 1; NARG, Nucleic Acids Research Group. shows that partially conjugated probes and/or those less than full-length are eluted first in capillary electrophoresis, followed by full-length probes with both reporter and quencher dyes. DHPLC (WAVE) analysis demonstrates the ability to resolve full-length probes based on hydrophobicity (Figure 3). WAVE produces baseline resolution of a full-length probe containing the reporter from the full-length oligonucleotide where the reporter coupling failed. The failed products eluted more ickly than the correct probe. Fluorescence monitoring verifies the position of the reporter on peak in each profile. Similar to capillary electrophoresis, the WAVE data demonstrate that a wellsynthesized crude probe has a profile similar to that of a purified probe. Secondly, the fluorescence traces illustrate the problem of TAMRA probes being contaminated with undesirable background fluorescence unless the probe is extensively purified. The probe with the most extensive purification procedure is NARG 21. It was purified by HPLC anion-exchange, followed by reverse phase HPLC. Importantly, NARG 21 is not substantially different in purity than the NARG 01 probe that was not purified postsynthesis, as judged by PAGE analysis (Figure 1), capillary electrophoresis analysis (Table 1), and DHPLC (Figure 3).

The results of the functional analysis by TaqMan assay are summarized in Table 1 . The mean of the $\Delta \mathrm{Rn}$ values

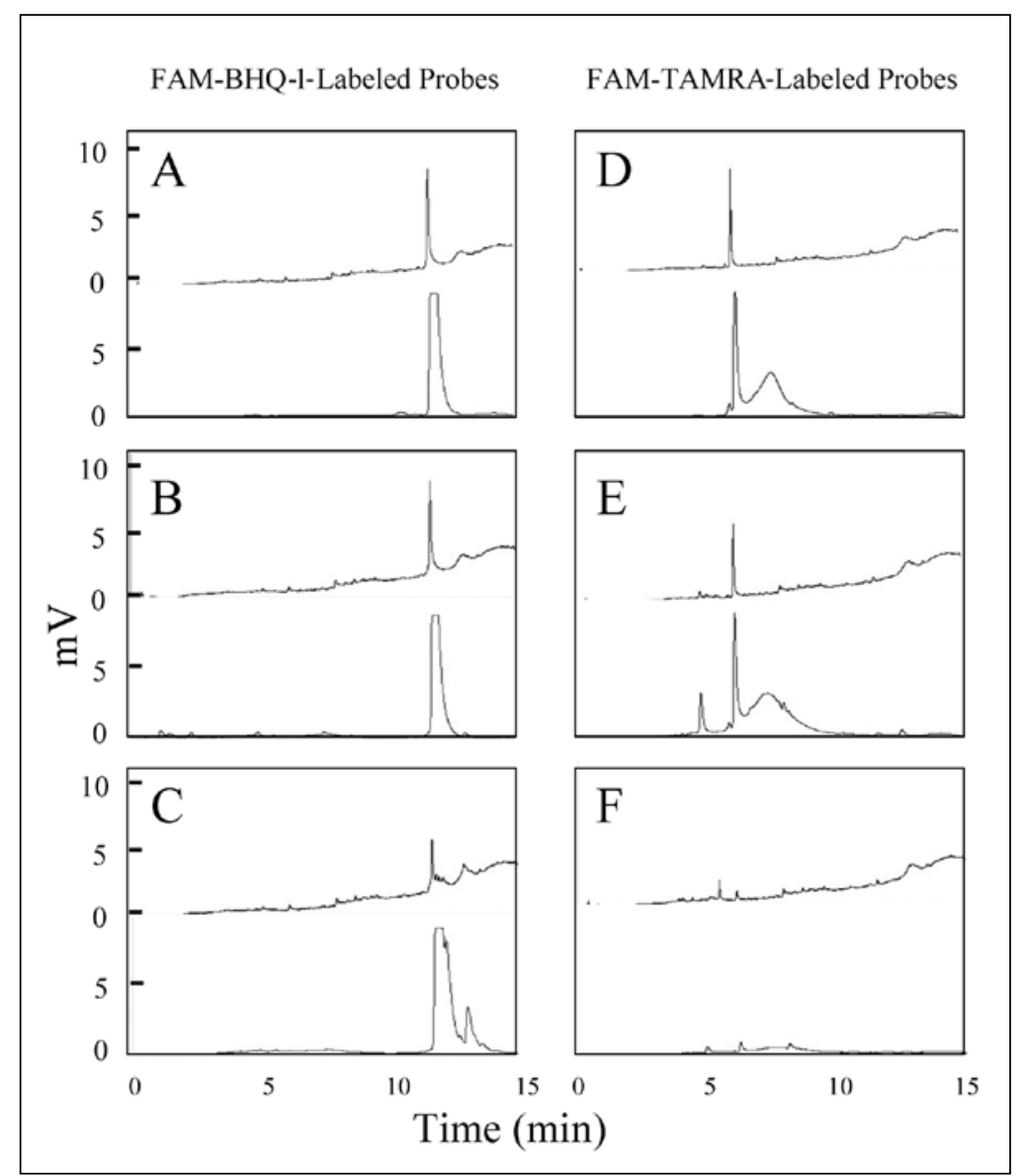

Figure 3. Resolution of probes by hydrophobicity utilizing denaturing high-performance liquid chromatography (DHPLC). The top curve and bottom curve of each panel are the UV $(260 \mathrm{~nm})$ traces and fluorescence traces (excitation, $496 \mathrm{~nm}$; emission, 520 $\mathrm{nm}$ ) of the HPLC elution, respectively. (A-C) BHQ-1-quenched probes. (D-F) TAMRAquenched probes. (A-F) Data as in Figure 2. Partially conjugated probes and/or those less than full-length came off first, followed by full-length probes with both reporter and quencher dyes. The correct BHQ-1 probe elutes at $12.5 \mathrm{~min}$. The correct TAMRA probe elutes at $6.7 \mathrm{~min}$. $\mathrm{mV}$, output of the UV or the fluorescence monitors in millivolts. BHQ-1, Black Hole Quencher 1. 
for all concentrations of the standard curve was calculated (Table 1, Mean $\Delta \mathrm{Rn})$. The $\mathrm{C}_{\mathrm{t}}$ value at 2000 copies of the standard, the $r^{2}$, slope, and y-intercept were reported for each probe. Additionally, the mean $\Delta \mathrm{Rn}$ and $\mathrm{C}_{\mathrm{t}}$ values for $50 \mathrm{ng}$ of human genomic DNA as template were reported. Thirty-three of the 35 probes gave remarkably similar results despite a broad range of purities from $99 \%$ down to only $18 \%$. Probes 24 and 25 gave insufficient or very low signal in real-time PCR due to extremely low reporter conjugation (Table 1).

Comparing purity values with those for several other measured parameters, such as the slope $(0.15)$, y-intercept (0.06), or $r^{2}$ values (0.07) for the standards, showed no significant correlation. However, when the purity values for the probes were compared to the $C_{t}$ values of the standard at 2000 copies, we found a correlation coefficient of 0.45 . There was an even greater correlation with the $\Delta \mathrm{Rn}\left(r^{2}=0.64\right)$. Similar results were obtained when $50 \mathrm{ng}$ of human genomic DNA were used as the template, although both the $\Delta \mathrm{Rn}$ and $\mathrm{C}_{\mathrm{t}}$ values were approximately $75 \%-80 \%$ of the values for the standard at 2000 copies (Table 1). In spite of the similarity in $\mathrm{C}_{\mathrm{t}}$, slope, or $\mathrm{y}$-intercept, the effect of the purity on the dynamic range of the fluorescent signal or $\Delta \mathrm{Rn}$ was significant (Figure 4). Figure 4 illustrates a comparison of one of the better crude BHQ-1 probes in terms of purity (NARG 01, 86.6\%) with a probe half as pure (NARG 22, 41.1\%). Probes with the highest purity showed a more robust dequenching of the reporter FAM signal with increasing cycles (Figure 4, $\mathrm{B}$ and $\mathrm{E})$. This results in a much higher final $\Delta \mathrm{Rn}$ value or signal-to-noise ratio in the later cycles (Figure 4, A and D). Interestingly, both of these probes produced standard curves with identical slopes and correlation coefficients (Figure 4, C and F). In general, higher purity probes gave lower $\mathrm{C}_{t} \mathrm{~s}$ and higher fluorescent signals $(\Delta R n)$ for a given amount of template. This is due to the more rapid accumulation of reporter signal at each cycle, reaching detectable levels in fewer PCR cycles. As the

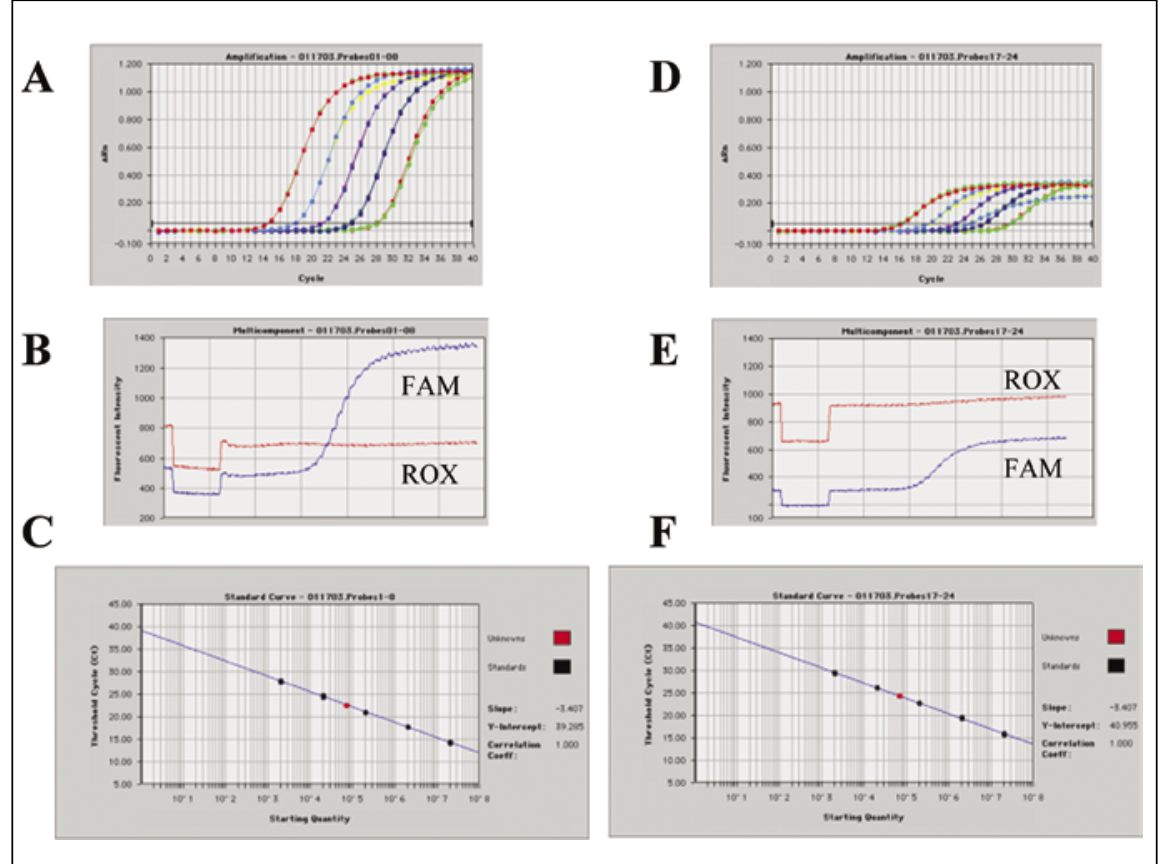

Figure 4. Comparison of the effects of good or poor probe synthesis on real-time PCR. (A-C) Probe NARG 01 from a good synthesis; 86.6\% purity (Table 1). (A) Amplification plot of all five standard dilutions, good ( $>1.0)$ difference between normalized reporter signal and background $(\Delta \mathrm{Rn})$ for all standards. (B) Raw fluorescence showing high fluorescent intensity of the FAM reporter compared to the ROX internal control dye. (C) Standard curve. (D-F) Probe NARG 22 from a poor synthesis; $41.1 \%$ purity (Table 1). (D) Amplification plot of all five standard dilutions, poor $(<0.4) \Delta R n$ for all standards. (E) Raw fluorescence showing weak initial signal and low fluorescent intensity of the FAM reporter compared to the ROX internal control dye. (F) Standard curve. NARG, Nucleic Acids Research Group.

purity of the probe decreases, the number of cycles it takes to reach threshold (significance above background) shifts to the right. This is due to the roughly 2-fold difference in the amplitude of the raw fluorescence signal, which can be seen in the amplification plot (Figure 4, $A$ and $D)$ in which there is a difference of approximately $2 \mathrm{C}_{\mathrm{t}} \mathrm{s}$ at every level of template for the high-purity probe versus the lower purity probe $(14.4,17.9$, $21.2,24.7,28.0$ versus $16.0,19.5,22.9$, 26.3, 29.6, respectively).

\section{DISCUSSION}

The results of this study show that very effective FAM/BHQ-1- or FAM/ TAMRA-quenched FRET probes can be synthesized without expensive and timeconsuming postsynthesis purification. One hundred percent of the respondents said that they found the synthesis easy. Of the 18 laboratories that participated in the study, 8 do not routinely synthesize this type of DNA. For the facilities that made these probes for the first time, all except one was successful.

An interesting observation was that the purity of the probe had no effect on the efficiency of the human $\beta$-actin assay. In the real-time PCR performance analysis, we were surprised that 33 of 35 probes resulted in standard curves with very similar slopes down to 2000 copies of standard template. This result would imply that assay efficiency is due primarily to how well the primer pairs work in PCR. The data show a well-synthesized crude probe (NARG 26, 91.8\% full-length probe) can perform comparably to a purified probe (NARG 13, 97.9\% full-length probe). Even a probe containing only $18.1 \%$ full-length probe (NARG 29) allowed for detection down to 2000 copies when used with an optimized primer pair on the oligonucleotide template (Table 1). Purer probes gave larger $\Delta R n s$, which resulted in a larger dynamic range. (Table 1) (Figure 4). However, neither the size nor quality of the template appears to have influenced the $\Delta \mathrm{Rn}$ because for all the probes tested, the $\Delta \mathrm{Rn}$ of the genomic DNA was about $75 \%$ that of the synthetic singlestranded oligonucleotide standard (calculated from Table 1). BHQ-1 probes 
are easier to synthesize than TAMRA probes. No changes in protocols or reagents are necessary to synthesize good-quality BHQ-1-quenched probes. TAMRA probes need milder deprotection conditions to prevent inactivation of the dye, thereby requiring the use of more expensive monomer bases compatible with the milder deprotection conditions. Nonpurified BHQ-1 probes tend to be of higher purity than the nonpurified TAMRA probes and produce higher $\Delta$ Rns. Several of the nonpurified BHQ-1-quenched probes approached the purity of the HPLC-purified probes. However, for the TAMRA-quenched probes, none of the crude probes was greater than $75 \%$ pure (Table 1). BHQ-1-quenched probes $>50 \%$ in full-length resulted in good (>0.8) $\Delta$ Rns. Moreover, BHQ-1quenched probes had a larger dynamic range $(\Delta \mathrm{Rn})$ than TAMRA-quenched probes (Table 1). The latter is most likely due to the more efficient quenching by BHQ- 1 versus TAMRA.

The cost of a commercially prepared dual-labeled probe at the 200-nmol scale is generally $\$ 200-\$ 400$. A consensus by this group estimates the cost of preparing a $200-\mathrm{nmol}$ scale probe at $\$ 50-\$ 70$ without post-synthesis purification. This study demonstrated that quality dual-labeled fluorescent probes suitable for real-time PCR can be made without purification at a price substantially lower than commercially purchased probes. This study also demonstrated that a variety of quality-control methods are adequate for assessing the quality of dual-labeled probe synthesis (Figures 1-3). The cost of the equipment for quality control may vary from $\$ 150,000$ for a mass spectrometer to less than $\$ 1000$ for a PAGE apparatus and a UV light.

In summary, the ease with which BHQ-1-quenched dual-labeled probes can be made in DNA synthesis core facilities and the absence of a need to purify them by HPLC prior to real-time PCR experiments should allow substantial cost savings in real-time PCR experiments. Moreover, this enables core facilities to deliver dual-labeled probes to the user within $24 \mathrm{~h}$ of placing an order. Thus, we recommend that core facilities focus their attention on perfecting their regular DNA synthe- sis and reporter coupling efficiencies. This will result in the production of the highest quality dual-labeled probes so that postsynthesis purification will be unnecessary. We further recommend that real-time PCR users concentrate on perfecting the design of the primers for the real-time PCR assays instead of on probe quality. It is our hope that real-time PCR experiments will now be as affordable and successful as other experiments that utilize unmodified oligonucleotides.

\section{ACKNOWLEDGMENTS}

We gratefully acknowledge all the participants of this study, especially those who do not make dual-labeled FRET probes on a regular basis and were brave enough to give them a try. We would like to acknowledge the hard work of Glen Miller and Emmanuelle Nicolas of the Fox Chase Cancer Center (FCCC), Josef Limor of the Centers for Disease Control and Prevention (CDC), and Ying Wang of the University of Texas Health Science Center (UTHSC)-Houston. Without their help and skill, this study would not have been possible. We would like to thank Kathleen Mills (Millennium Pharmaceutical), Stephen Scaringe (Dharmacon Research), and Gregory A. Buck (Virginia Commonwealth University) for their assistance in the preliminary studies. We would also like to thank our Association of Biomolecular Resource Facilities (ABRF) Executive Board ad hoc, Dr. Susan Hardin of the University of Houston, for her guidance.

\section{REFERENCES}

1.Heid, C.A., J. Stevens, K.J. Livak, and P.M. Williams. 1996. Real time quantitative PCR. Genome Res. 6:986-994.

2.Gibson, U.E.M., C.A. Heid, and P.M. Williams. 1996. A novel method of real time quantitative RT-PCR. Genome Res. 6:9951001.

3.Lakowicz, J.R. 1983. Energy transfer, p. 303-339. In Principles of Fluorescent Spectroscopy. Plenum Press, New York.

4.Lyamichev, V., M.D. Brow, and J.E. Dahlberg. 1993. Structure-specific endonucleolytic cleavage of nucleic acids by eubacterial DNA polymerases, Science 260:778-783.

5.MacKay, I.M., K.E. Arden, and A. Nitsche. 2002. Real-time PCR in virology. Nucleic
Acids Res. 30:1292-1305.

6.Bustin, S.A. 2000. Absolute quantification of mRNA using real-time reverse transcription polymerase chain reaction assays. J. Mol. Endocrin. 25:169-193.

7.Bustin, S.A. 2002. Quantification of mRNA using real-time reverse transcription PCR (RT-PCR): trends and problems. J. Mol. Endocrin. 29:23-39.

8.Zhu,Y.F., C.N. Chung, N.I. Taranenko, S.L. Allman, S.A. Martin, L. Haff, and C.H. Chen. 1996. The study of 2,3,4-trihydroxyacetophenone and 2,4,6-trihydroxyacetophenone as matrices for DNA detection in matrix-assisted laser desorption/ionization time-of-flight mass spectrometry. Rapid Commun. Mass Spectrom. 10:383-388.

Received 15 October 2003; accepted 3 November 2003.

\author{
Address correspondence to: \\ Anthony T. Yeung \\ Fox Chase Cancer Center \\ 333 Cottman Avenue \\ Philadelphia, PA 19111-2497, USA \\ e-mail: anthony.yeung@fccc.edu
}

